\title{
Maternal Antimüllerian Hormone as a Predictor of Euploid Is Independently Associated With Cumulative Live Birth Rates
}

\author{
Xiaoling Wu \\ Zhengzhou University First Affiliated Hospital \\ Huixia Zhang \\ Zhengzhou University First Affiliated Hospital \\ Wenbin Niu \\ Zhengzhou University First Affiliated Hospital \\ Jiawei Xu \\ Zhengzhou University First Affiliated Hospital \\ Haixia Jin \\ Zhengzhou University First Affiliated Hospital \\ Gang Li ( $\square$ lgvigor@126.com ) \\ Zhengzhou University First Affiliated Hospital
}

\section{Research}

Keywords: AMH, euploid marker, oocyte quality, ovarian reserve, live birth rate

Posted Date: September 2nd, 2021

DOI: https://doi.org/10.21203/rs.3.rs-806129/v1

License: (c) (i) This work is licensed under a Creative Commons Attribution 4.0 International License. Read Full License 


\section{Abstract}

Background: Serum antimÜllerian hormone (AMH) level has been reported to be associated with pregnancy rates after assisted reproduction. It is unclear whether AMH levels affect number of euploid blastocysts and cumulative live birth rates (CLBR). The aim of this study was to investigate whether AMH levels are associated with cumulative live birth rates (CLBR) through their relationship with oocyte quality.

Methods: A total of 975 consecutive infertile women undergoing 1825 preimplantation genetic screening (PGS) analysis. Serum AMH levels were measured by AMH Gen II assay kit within the 3 months before Controlled Ovarian Hyperstimulation. Embryos were cultured and biopsied at the blastocyst stage.

Results: Among 975 women undergoing PGS analysis. Age, serum AMH and number of oocytes retrieved were significantly and independently related to number of euploid blastocysts available for patients to transfer $(P<.001)$. in patients displaying superior serum AMH levels, cumulative live birth rates (CLBR) were significantly increased independently from age and number of oocytes retrieved $(P<.001)$, and this group with lower AMH levels also showed increased pregnancy loss rates.

Conclusions: Serum AMH levels are positively age-independent associated with cumulative live birth rates after all viable embryos are transferred from the first ovarian stimulation cycle. And embryonic euploidy outcome was superior in patients with higher AMH levels. These present findings confirm that serum AMH levels might reflect not only ovarian reserve but also qualitative aspects of oocytes, which will effect the clinical application of PGS.

\section{Introduction}

Aneuploidy is an abnormal karyotype of which chromosome number is not divisible by 23 , commonly referred to the absence or addition of one or more complete chromosomes or partial chromosome fragments in the chromosome set. It is mainly formed during the process of meiosis or mitosis that chromosomes are nondisjunction, or early/delayed [1]. Aneuploidy can lead to low fetal intelligence, gender abnormalities, spontaneous abortion and a variety of deformities. According to the data statistics demonstrated that aneuploidy is the most common chromosomal abnormality in IVF-ET cycles embryos, affecting more than 50\% of human embryos, which is the main cause of embryo implantation failure and abortions in IVE-ET cycle [2,3]. Thus, screening for aneuploidy in couples at high aneuploidy risk is one of the main contents of PGS

Nowadays, the age-associated increase in aneuploidy rate of the embryo due to the rising chromosomal abnormalities in the oocytes has been well established [4, 5].Age as an independent and significant factor affecting the outcome of assisted reproductive technology [6]. It is well recognized that female age is associated with the occurrence of aneuploidy, the increased female age significantly reduces the probability of obtaining at least one euploidy embryo per treatment cycle [5, 7]. The decline in fertility in women is associated with age-related follicular aging, and ovarian primordial follicles are age-related reduced through recruitment, ovulation and atresia, clearly decreased ovarian reserve and exhausted female reproductive function $[8,9]$. There is still doubt as to whether the decline in oocyte quality is directly related to the decrease in ovarian reserve.

Ovarian reserve can now be accurately estimated by measuring the value of baseline serum AMH. AntimÜllerian hormone is a member of the TGF- super family, which is a single reliable indicator of ovarian reserve, regardless of the menstrual cycle [10,11]. In fact, numerous studies have found that serum AMH level is related to the number of fetal aneuploidy rate [12]. Such studies have shown that the risk of embryonic aneuploidy is mainly due to changes in advanced age and associated oocytes, rather than changes in ovarian reserve in isolation [13]. However, the possible relationship between serum AMH level and oocyte quality remains to be further studied. The occurrence of clinical live birth can indirectly reflects oocyte/embryo quality. Female fertility peaks at 2030 [14]; after 30, fertility declines gradually; after 35, fertility declines more significantly [5]. Similarly, serum AMH levels drop sharply after 35 years of age. Therefore, we make the assumption that AMH reflects the process of follicular aging [15]. Thus, the present study was performed to investigate whether the level of serum AMH has a beneficial effect on cumulative live birth rates in PGS cycles, discussing whether the results may reflect the quality of oocytes.

\section{Materials And Methods}




\section{Study subjects}

Data in this retrospective cohort study were from the Clinical Reproductive Medicine Management System/Electronic Medical Record Cohort Database (CCRM/EMRCD) in Reproductive Medical Center, First Affiliated Hospital of Zhengzhou University. The data of each cycle contains demographics, ART history, protocol used and embryonic outcomes, collected from 4616 PGT cycles, performed between December 2016 and July 2020. Inclusion criteria were: 1) advanced maternal age; 2) repeated spontaneous miscarriage for unknown reasons; 3) repeated planting failure for unknown reasons. Exclusion criteria were: 1) uterine abnormalities that could interfere with further embryo development; 2) patients with systemic diseases or endocrine disease (PCOS and thyroid disease), including autoimmune disorders; 3 ) cycles that were cancelled caused incompleted clinical data; 4) infertility caused by male factors.

\section{Stimulation protocol}

In the mid-luteal phase before the stimulation cycle. The long GnRH agonist protocol was used for controlled ovarian stimulation. The starting dose of $\mathrm{rFSH}$ was individually based on patient age, body mass index (BMI), AMH levels, day-3 FSH and antral follicle count, ranging from 100-225IU/d., Patients were monitored with ovarian ultrasound and gonadotropin doses were adjusted according to patient's response to stimulation. When two or more mature follicles had reached a diameter $\geq 18 \mathrm{~mm}$, oocyte maturation was administered with recombinant human chorionic gonadotropin (HCG) 1000IU. 34-36 hours later, transvaginal ultrasound- guided oocyte retrieval was performed.

\section{Insemination, embryo culture and biopsy procedure}

All Oocyte insemination procedures were conducted by ICSI for all PGS cycles. The source of male semen was masturbation and superior sperm was selected by Percoll gradient centrifugation and Swim-up. Retrievaled oocytes surrounded by the granulose cells was incubated for 3-4 hours. The granulose cells were blown off with a plastic pipette (Flexipet; 170 and 140 mm inner diameters; Cook), then mature oocytes (MII stage) with obvious extrusion of the first polar body were subjected to intracytoplasmic sperm injection immediately after denuding. The sperm-injected oocytes were transferred to a single drop of cleavage medium (Quinn Advantage Protein Plus Cleavage Medium; Sage) under oil-soluble conditions of $37^{\circ} \mathrm{C}, 5 \% \mathrm{O} 2$ and $6 \% \mathrm{CO} 2.3$ days after insemination, all developing embryos were converted to medium-transformed sequential culture and a noncontact 1.48-um diode laser was used to form a 6-9um diameter circular opening in the zona pellucida to allow the trophectoderm cells to herniate. Embryos were cultured to the blastocyst stage on the 5th to 7th day after insemination to be performed biopsy. On the day of biopsy, 5-10 trophectoderm cells were gently aspirated into a biopsy pipette(cook) and separated from the blastocysts. The obtained trophectoderm cells were washed in sterile phosphate-buffered saline solution (PBS), then transferred in microcentrifuge PCR tubes containing $2 \mathrm{~mL}$ PBS, spun down for a few seconds, and sent to Genoma laboratory for analysis, as previously described elsewhere[5]. Then blastocysts after biopsy were cryopreserved immediately by vitrification freezing technology. After the embryo genetic analysis was completed, the embryos with normal results were selected for resuscitation transplantation.

\section{preimplantation genetic screening}

The whole-genome amplification (WGA) technique was utilized to enlarge the whole- genome single-copy DNA in a single cell from the pg-level amplification to the ug level. Subsequently, aneuploidy was performed by NGS, using a high-throughput sequencer NextSeq CN500 (China Berry and Kang) for sequencing. Results of PGS were recorded as euploid, aneuploid, or mosaic. The embryo without the deletion and/or repetition is an euploid embryo and is judged to be an available embryo.

\section{Hormonal assays}

All the selected infertile women did not undergoing any hormone therapy in the 3 months prior to initiating ovarian stimulation. Before treatment all patients had a blood sample taken for analysis of serum hormone on day 3 to day 5 of menstruation: Two tubes of peripheral venous blood were taken at 8-10 am after 8 hours of fasting. One tube of Serum AMH was measured by AMH enzyme immunoassay Gen II (Beckman Coulter), strictly following the manufacturer's instructions. The analytical sensitively of the ELISA assay was estimated to be $0.1 \mathrm{ng} / \mathrm{mL}$. The measurement was repeated 3 times to make the observation more reliable.

\section{Clinical outcome follow-up}


Serum $\beta$-hCG values were measured 14 days after embryo transfer, and luteal support drugs were discontinued if not pregnant. After 4-5 weeks of transplantation, vaginal ultrasonography was performed. The pregnancy and fetal heartbeat sac were found in the uterine cavity. The corpus luteum support drug was gradually reduced to 8-10 weeks after transplantation. A clinical pregnancy was defined as the ultrasounddetected presence of one or more gestational sacs at 7 weeks of amenorrhea. Miscarriage was defined as intrauterine pregnancy loss before 24 weeks of gestation. CLBR was defined as the percentage of live births per woman during the first complete stimulation cycle.

\section{Definition of Age and AMH Groups}

For reducing possible biases affecting the relationship between serum AMH levels and PGS outcome, particularly patient's age, the study population (1825 COS cycles) was divided into different age and AMH groups. First, according to the upper and the lower tertiles of female ages, three groups were defined: $\leq 34$ years( 490 cases), $35-38$ years ( 240 cases) and $\geq 39$ years (245 cases).Moreover, three AMH groups were established according to the 25 th $(1.838 \mathrm{ng} / \mathrm{ml})$ and the 75 th $(4.523 \mathrm{ng} / \mathrm{ml})$ percentiles of the study population as follows: low AMH ( $\leq 1.838 \mathrm{ng} / \mathrm{mL} ; 245$ cases); medium AMH (1.838-4.523 ng/mL; $485 \mathrm{cases})$; and high $\mathrm{AMH}(\geq 4.523 \mathrm{ng} / \mathrm{mL} ; 245$ cases$)$.

\section{Statistical analysis}

Statistical analysis was performed using the IBM SPSS Statistics 24.0 software. Kolmogorov-Smirnov test was used to assess continuous variables. Data were compared between three groups, Subgroup analysis was performed stratified according to age. To identify characteristics that may be associated with the number of euploid blastocysts, multivariate logistic regression analysis was performed: Univariate regression analyses were performed to identify factors that predict the number of euploid blastocysts, Variables found to have tendency of association with the primary outcome $(P<0.2)$ in the univariate analysis were included in the multivariate analysis. And binary logistic regression was conducted to verify whether AMH levels were associated with birth outcome, independently from confounding factors, particularly age and the number of oocytes retrieved. A $P$-value $<0.05$ was considered to be statistically significant.

\section{Results}

\section{Baseline Characteristics}

A total of 1825 IVF cycles of 975 patients were obtained for study. There were 590 patients underwent IVF-ET owing to recurrent spontaneous miscarriage (RSM). The mean age was $34.7 \pm 5.3$ ( range $21-47$ years). AMH was log-normally distributed with a mean \pm SD of $3.61 \pm 2.95$ (range $0.14-23 \mathrm{ng} / \mathrm{mL}$ ). The mean BMI was $23 \pm 2.7\left(\right.$ range $\left.16.4-31.4 \mathrm{~kg} / \mathrm{m}^{2}\right)$. The total number of blastocysts available to the analysis was 4895, and 1660(34\%) of them were euploid after PGS analysis. The occurrence of birth was 515(52.8\%).

\section{Patients Characteristics and IVF-ET Data between AMH Groups}

Patients Characteristics and IVF-ET data in the different age and AMH groups are presented in Table 1. Age, AMH and embryonic euploidy rate and so on were compared between three groups. Subgroup analysis was performed stratified according to age. We observed a stepwise increase in the number of oocytes retrieved of patients from the low to the high AMH groups $(\leq 34$ y: L: 10; M: 17; H: 23 versus 35-38 y: L: 8; M: 16; H: 21 versus $\geq 39$ y: L: 7; M: 14; H: 20, respectively, $P<0.05)$. This progressive pattern was reproduced in the three age groups, indicating that the relationship between serum AMH levels and IVF-ET outcome possibly is not influenced by age. As expected, the number of blastcysts per patients undergoing biopsy was significantly increased in the high AMH group compared with the medium and low AMH groups( 34 y: L: 4; M: 5; H: 8.5; 35-38 y: L:3; M: 4; H: 6; $\geq 39$ y: L: 2; M: 1; H: 5 , respectively, $P<0.05)$. Furthermore, the prevalence of euploidy, which is putatively associated to the success of IVF-ET cycles, did not vary significantly in the medium and high AMH groups in the patients $\geq 39$ years $(P>0.05)$, which probably reflects the strong association between female age and ovarian quality. We also observed a stepwise increase in cumulative clinical pregnancy, ongoing pregnancy rates from the low to the high AMH groups, which indicated that the relationship between AMH with PGS outcome possibly is not influenced by age. As expected, in the low AMH group, pregnancy losses were more frequent than in the medium and high AMH groups.In addition, our data confirmed that serum AMH levels were quantitatively associated with oocyte and embryo availability. 


\section{Biopsy results}

To evaluate the relationship between $\mathrm{AMH}$ and the number of euploid blastocysts, multiple linear regression was performed, controlling for covariates including age, $\mathrm{BMI}, \mathrm{AMH}$, number of oocytes retrieved, and number of embryos biopsied. All variables were entered in Multiple linear regression at a level of $P<0.2$. Results are reported as slope (b), 95\% confidence interval $(\mathrm{Cl})$ and $P$ value. Multiple regression analysis showed that female age $\llbracket$ serum $\mathrm{AMH}$ and number of oocytes retriened, were independent predictors of the number of euploid embryos in IVF $\operatorname{cycles}(P<0.05)$. For these three variables, analysis of variance showed a statistically significant effect in determining the number of euploid blastocysts per patient $(P<0.05)$; No significant association was found between the number of euploidy and other patient or cycle characteristics, such as body mass index, protocol, type and day of blastocyst biopsy $(P>0.05)$. Finally, to measure the effect size of this association, the number of euploid blastocyst available for transfer was chosen as outcome. The complete ultivariate model was as follows: $y=4.099+0.147^{\star} \mathrm{AMH}-0.115^{\star}$ female age + $0.069 *$ the number of oocytes retrieved $\left(R^{2}=0.377\right.$; adjusted $R^{2}=0.367 ; \mathrm{VIF}<10 ; \mathrm{F}$ ratio $\left.=38.54, P<0.000\right)($ Table 2$)$. This indicates that for two women of similar age and oocytes retrieved, higher serum AMH lead to increased number of euploid blastocyst for embryo transfer.

\section{Live Birth Outome}

Live birth rates in the different age and AMH groups also were presented in Supplemental Fig. 1. Baseline demographics and cycle characteristics were compared between patients who did and did not achieve live birth. In order to compare the influence of various factors, Stratification was used in the data of factors including age, $\mathrm{AMH}$, frozen embryo transfer (FET) cycles and the number of oocytes retrieved. Controlling for age, the medium level of serum AMH impacted the odds of achieving live birth compared to low level $(\mathrm{OR}=0.27 ; 95 \% \mathrm{Cl}: 0.09-0.77 ; P=0.014)$, the high level of serum $\mathrm{AMH}$ impact the odds of achieving live birth $(\mathrm{OR}=0.48 ; 95 \%$ $\mathrm{Cl}: 0.22-1.04 ; P=0.063)$. Accounting for the same covariates, tha same as the number of FET cycles $(\mathrm{OR}=1.66 ; 95 \% \mathrm{Cl}$ : $0.43-6.38$; $P=0.034)$ or female age $(\mathrm{OR}=1.91 ; 95 \% \mathrm{Cl}: 0.88-4.16 ; P=0.013))$. The number of oocytes retrieves did not impact the odds of live birth $(\mathrm{OR}=0.49 ; 95 \% \mathrm{Cl}: 0.22-1.10 ; P=0.67)$. ( Fig. 1$)$

\section{Discussion}

Our study is clearly to investigate the independent relationships between serum AMH levels and cumulative live birth rates of infertile women underwent euploidy transfer from the first ovarian stimulation cycle in PGS analysis. After adjusting for potential confounders, we found that live birth outcome is superior in women with relatively higher serum AMH level. Similarly, independently from age, increased value of $\mathrm{AMH}$ is associated with increased number of euploidy blastocysts.

\section{AMH and Number of Euploidy Blastocysts}

It has been previously shown that $\mathrm{AMH}$ is considered to have high predictive accuracy for ovarian response and oocyte yield after ovarian stimulation, suggesting that AMH could be an excellent predictor of quantitative aspects of ART [16, 17]. In our study, women who attained more euploidy blastocysts was found to have increased serum AMH concentration. This was in line with findings by Antonio La Marca et al. which indicates that independently from age, increased ovarian reserve is in accordance with an increased rate of embryo aneuploidy $[5,18,19]$. In addition, This conclusion could explain that after a unilateral oophorectomy (reduced ovarian reserve) in female mice, the number of aneuploidy (low-quality oocytes) will rise earlier than expected [20].

\section{AMH and Cumulative Clinical Live Birth Rates}

In the clinical practice, the availability of euploid blastocysts for patients is extremely important. According to our findings, in women with the same ovarian reserve, old age may decrease the number of aneuploidy blastocysts obtained, leading to an extremely poor outcome with IVF. This scientific background suggested a strong correlation between female aging and decreased ovarian quality. This is in line with the previous study [5, 21-23]. AMH levels and the reproductive competence of the oocyte remains to be further investigated. We sought to examine live birth rates after COS for IVF-ET carried out in patients with different $\mathrm{AMH}$ levels. For reaching this objective, the presumable influence of age on the relationship between serum AMH levels and live birth rates was excluded by means of both age group stratification and multivariate analysis. We observed that serum AMH levels were associated with live birth rates after IVF-ET and that such a relationship was not explained by usual fluctuations of age and oocyte yield, as confirmed by multivariate regression analysis. Therefore, we can make the assumption that the ovarian reserve 
decline has not only a quantitative((decreased number of primordial follicles), but also a qualitative effect (low quality oocytes and increased embryo aneuploidy risk),leading to pregnancy failure in IVF-ET cycles. This makes clinical management more difficult for patients with low levels of AMH, as the number of embryos available for PGS drops sharply. In theory, PGS should be able to block the risk of oocyte and embryo aneuploidy caused by advanced age, and improve the clinical outcome of elderly women by increasing the embryo implantation rate and reducing the abortion rate.At the same time, it greatly reduces the mental and economic trauma caused by repeated abortion or induced labor caused by prenatal diagnosis. However, in clinical practice, euploid blastocysts in patients with too low AMH transplantation may still lead to implantation failure. This may be because low AMH not only resulted in low quality eggs, but also resulted in poor luteal quality and insufficient progesterone secretion. Therefore, the efficacy of PGS in elderly infertile women, especially in patients with very low AMH, is controversial. in which case PGS may cause some rare embryos with normal potential to fail to develop to blastocyst stage in vitro and be abandoned. Whether PGS can improve clinical outcomes in elderly women still needs to be further verified in a multi-center, large-sample, prospective randomized controlled clinical trial.

\section{AMH Reflects Oocyte Yield and Quality}

Our study suggests that a direct relationship may exist between $\mathrm{AMH}$ production and oocyte quality. In our study, AMH remained independently associated with CCPR after adjustment for age and oocyte yield. As a matter of fact, there are emerging evidence shows relationships between oocyte quantity and quality. An earlier study suggested that when the number of primordial follicles is below 25,000 thresholds, the rate of follicular depletion will increase substantially [24]. This number is reached at an average of 37.5 years in people, leading to a cliff-like decline of women fertility. Similarly, large-scale studies of women pursuing preimplantation genetic testing for aneuploidy (PGT-A) demonstrate that aneuploidy sharply rises beginning at age 38. Numerous studies have indicated that the decline of follicles stored in the female follicle pool can also affect the quality of the oocytes released by women [25-27]. In line with this, a study reported that cultured granulosa cells produced more AMH in the presence of oocytes [28].

In conclusion, this retrospective study demonstrates that independently from age, decreased ovarian reserve may lead to reduced rate of live birth after euploid embryo transfer. And embryonic euploidy outcome was superior in patients with higher AMH levels. These present findings confirm that serum AMH levels might reflect not only ovarian reserve but also qualitative aspects of oocytes, suggesting a direct relation between oocyte qualitative and quantitative aspect. The findings demonstrates that low concentration of $\mathrm{AMH}$ reduce not only oocyte yield but also the opportunity to obtain a great quality oocyte. This confirms a high correlation with the use of $\mathrm{AMH}$ to measure ovarian reserve in consultation with infertility patients.

Strengths and Limitations: The strength of the present study is that euploidy was selected as the outcome indicator, because the evaluation of embryo quality based on morphology has its limitation $[29,30]$. Another strong point is the endometrial preparation and synchronization to maximize endometrial function and receptivity. Moreover, male factors were excluded. The major weakness of the present study is the retrospective design with a small sample size, which needs further confirmation from large-sample prospective study.

\section{Abbreviations}

AMH: Anti-mÜllerian hormone; PGS: Preimplantation genetic screening; AFC: Antral follicle count; CLBR: Cumulative live birth rate; OPR: Ongoing pregnancy rate; BMI: Body mass index

\section{Declarations}

Acknowledgments: The authors thank for the women who participated in this study and all the physicians and nurses at the Reproductive Medicine Centre, the First Affiliated Hospital of Zhengzhou University, China, for their support in collecting the data.

Authors' contributions: Gang li conceived and designed the study, Xiaoling Wu and Huixia Zhang performed experimental analysis, statistical analysis and wrote the manuscript. Jiawei Xu and Haixia Jinconceived the study and revised the manucscript. All authors read and approved the final manuscript.

Authors' information: Not applicable. 
Availability of data and materials: The data are available from the corresponding author on reasonable request.

Ethical approval: This study was approved by the Ethics Committee of Reproductive Medicine Center, the First Affiliated Hospital of Zhengzhou University, China. The written informed consent was provided for the research purpose.

Consent for publication: Not applicable.

Competing interests: None.

Funding: This work was supported by the National Natural Science Foundation of China (81771534 to G. L., 81820108016 to Y. S.), National Key R\&D Program of China (2019YFA0110900 to Y. S.), Key science and technology foundation of Henan Province (172102310082 to G. L.), Key research projects of Henan higher education institutions (18A320057 to G. L.), Research and Development Project of Young Doctors in Reproductive Medicine of Chinese Medical Association (18010240753 to G. L.).

\section{References}

1. Carmen R, Lorena R, Amparo M, Emilia M, Pilar B, Tugce P, Thamara V: Impact of chromosomal abnormalities on preimplantation embryo development. Prenat Diagn 2010, 27:748-756.

2. Macklon NS, Geraedts JPM, Fauser BC: Conception to ongoing pregnancy: the 'black box' of early pregnancy loss. Hum Reprod Update 2002, 8:333-343.

3. Voullaire L, Collins V, Callaghan T, McBain J, Williamson R, Wilton L: High incidence of complex chromosome abnormality in cleavage embryos from patients with repeated implantation failure. Fertil Steril 2007, 87:1053-1058.

4. Morin SJ, Patounakis G, Juneau CR, Neal SA, Scott RT: Diminished ovarian reserve and poor response to stimulation in patients $<38$ years old: a quantitative but not qualitative reduction in performance. Hum Reprod 2018.

5. La Marca A, Minasi MG, Sighinolfi G, Greco P, Argento C, Grisendi V, Fiorentino F, Greco E: Female age, serum antimullerian hormone level, and number of oocytes affect the rate and number of euploid blastocysts in in vitro fertilization/intracytoplasmic sperm injection cycles. Fertil Steril 2017, 108:777-783 e772.

6. Menken J, Trussell J, Larsen U: Age and infertility. science 1986, 233:1389-1394.

7. Volarcik K, Sheean L, Goldfarb J, Woods L, Abdul-Karim FW, Hunt P: The meiotic competence of in-vitro matured human oocytes is influenced by donor age: evidence that folliculogenesis is compromised in the reproductively aged ovary. Hum Reprod 1998, 13:154-160.

8. Franasiak JM, Forman EJ, Hong KH, Werner MD, Upham KM, Treff NR: The nature of aneuploidy with increasing age of the female partner: a review of 15,169 consecutive trophectoderm biopsies evaluated with comprehensive chromosomal screening. Fertil Steril 2014, 101:656-663.e651.

9. Basille C, Frydman R, Aly AE, Hesters L, Fanchin R, Tachdjian G, Steffann J, Le Lorc'H M, Achour Frydman N: Preimplantation genetic diagnosis: state of the art. Eur J Obstet Gyneco/ 2009, 145:9-13.

10. Sills ES, Li X, Frederick JL, Khoury CD, Potter DA: Determining parental origin of embryo aneuploidy: analysis of genetic error observed in $\mathbf{3 0 5}$ embryos derived from anonymous donor oocyte IVF cycles. Mol Cytogenet 2014, 7:68.

11. Pellestor F, Andréo B, Arnal F, Humeau C, Demaille J: Maternal aging and chromosomal abnormalities: new data drawn from in vitro unfertilized human oocytes. Hum Genet 2003, 112:195-203.

12. Broer SL, Van Disseldorp J, Broeze KA, Dolleman M, Brent CO, Bossuyt P, Eijkemans MJC, Mol BWJ, Broekmans FJM: Added value of ovarian reserve testing on patient characteristics in the prediction of ovarian response and ongoing pregnancy: an individual patient data approach. Hum Reprod Update 2013, 19:26-36.

13. xiao J, yan Jh, yan S, mei S: Low anti-Mullerian hormone concentration is associated with increased risk of embryonic aneuploidy in women of advanced age. Reprod Biomed Online 2018, 37:178-183.

14. Cook CL, Siow Y, Taylor S, Fallat ME: Serum müllerian-inhibiting substance levels during normal menstrual cycles. Fertil Steril 2000, 73:859-861.

15. Meczekalski B, Czyzyk A, Kunicki M, Podfigurna-Stopa A, Pluciennik L, Jakiel G, Maciejewska-Jeske M, Lukaszuk K: Fertility in women of late reproductive age: the role of serum anti-Müllerian hormone (AMH) levels in its assessment. Journal of 
Endocrinological Investigation 2016, 39:1259-1265.

16. Broer SL, Dólleman M, Opmeer BC, Fauser BC, Mol BW, Broekmans FJM: AMH and AFC as predictors of excessive response in controlled ovarian hyperstimulation: a meta-analysis. Hum Reprod Update 2011, 17:46-54.

17. Tal R, Seifer DB, Wantman E, Baker V, Tal O: Antimullerian hormone as a predictor of live birth following assisted reproduction: an analysis of 85,062 fresh and thawed cycles from the Society for Assisted Reproductive Technology Clinic Outcome Reporting System database for 2012-2013. Fertil Steril 2018, 109:258-265.

18. Brodin T, Hadziosmanovic N, Berglund L, Olovsson M, Holte J: Antimullerian hormone levels are strongly associated with livebirth rates after assisted reproduction. J Clin Endocrinol Metab 2013, 98:1107-1114.

19. Thorne J, Loza A, Kaye L, Nulsen J, Benadiva C, Grow D, Engmann L: Euploidy rates between cycles triggered with gonadotropin-releasing hormone agonist and human chorionic gonadotropin. Fertil Steril 2019, 112:258-265.

20. Brook JD, Gosden RG, Chandley AC: Maternal ageing and aneuploid embryos-evidence from the mouse that biological and not chronological age is the important influence. Hum Genet 1984, 66:41-45.

21. Lee MS, Tzeng SL, Yang SF, Lin YP, Cheng EH, Huang CC, Yang YS, Lee TH: Correlation of serum anti-Mullerian hormone to follicular follicle stimulating hormone and implantation potential of the ensuing embryos. Clin Chim Acta 2017, 471:327-333.

22. Kunicki M, Skowronska P, Pastuszek E, Jakiel G, Smolarczyk R, Lukaszuk K: Do serum androgens influence blastocysts ploidy in karyotypically normal women? Syst Biol Reprod Med 2019, 65:281-287.

23. Ligon S, Lustik M, Levy G, Pier B: Low antimullerian hormone (AMH) is associated with decreased live birth after in vitro fertilization when follicle-stimulating hormone and AMH are discordant. Fertil Steril 2019, 112:73-81 e71.

24. Gougeon A, Ecochard R, Thalabard JC: Age-related changes of the population of human ovarian follicles: increase in the disappearance rate of non-growing and early-growing follicles in aging women. Biol Reprod 1994, 50:653-663.

25. Holte J, Brodin T, Berglund L, Hadziosmanovic N, Olovsson M, Bergh T: Antral follicle counts are strongly associated with livebirth rates after assisted reproduction, with superior treatment outcome in women with polycystic ovaries. Fertil Steril 2011, 96:594-599.

26. van Noord-Zaadstra BM, Looman CW, Alsbach H, Habbema JD, te Velde ER, Karbaat J: Delaying childbearing: effect of age on fecundity and outcome of pregnancy. BMJ 1991, 302:1361-1365.

27. Grande M, Borobio V, Bennasar M, Stergiotou I, Mercade I, Masoller N, Penarrubia J, Borrell A: Role of ovarian reserve markers, antimullerian hormone and antral follicle count, as aneuploidy markers in ongoing pregnancies and miscarriages. Fertil Steril 2015, 103:1221-1227 e1222.

28. Salmon NA, Handyside AH, Joyce IM: Oocyte regulation of anti-Müllerian hormone expression in granulosa cells during ovarian follicle development in mice. Dev Biol 2004, 266:201-208.

29. de Boer KA, Catt JW, Jansen RPS, Don L, Steven M: Moving to blastocyst biopsy for preimplantation genetic diagnosis and single embryo transfer at Sydney IVF. Fertil Steril 2004, 82:295-298.

30. FrancOis A, Wilson RD, Allen V, Blight C: Preimplantation genetic testing. J Assist Reprod Genet 2009, 31:761-775.

\section{Tables}


Table 1

Patient characteristics and IVF-ET data in the different AMH and age groups $(n=975)$

\begin{tabular}{|c|c|c|c|c|c|c|c|c|c|}
\hline \multirow{3}{*}{ Variable } & \multicolumn{3}{|c|}{$\leq 34$ y $(n=495)$} & \multicolumn{3}{|c|}{$35-38$ y $(n=240)$} & \multicolumn{3}{|c|}{$\geq 39$ y $(n=245)$} \\
\hline & Low & medium & high & low & medium & High & low & medium & high \\
\hline & $\mathrm{AMH}$ & $\mathrm{AMH}$ & $\mathrm{AMH}$ & $\mathrm{AMH}$ & $\mathrm{AMH}$ & $\mathrm{AMH}$ & $\mathrm{AMH}$ & $\mathrm{AMH}$ & $\mathrm{AMH}$ \\
\hline $\mathrm{n}$ & 85 & 220 & 185 & 50 & 160 & 30 & 110 & 105 & 30 \\
\hline Age, y & $31 \pm 1.8$ & $31 \pm 2.9$ & $30 \pm 3.3$ & $37 \pm 0.9$ & $37 \pm 1.1$ & $36 \pm 0.6$ & $42 \pm 1.4$ & $41 \pm 2.8$ & $41 \pm 0.8$ \\
\hline $\mathrm{BMI}, \mathrm{kg} / \mathrm{m}^{2}$ & $22.0 \pm 3.1$ & $23.4 \pm 3.2$ & $22.6 \pm 5.4$ & $23.5 \pm 1.8$ & $23.6 \pm 2.3$ & $23.5 \pm 1.8$ & $22.6 \pm 2.6$ & $23.1 \pm 3.1$ & $23.4 \pm 4.2$ \\
\hline D3 FSH & $7.86 \pm 0.8$ & $7.11 \pm 0.1$ & $6.48 \pm 0.2$ & $8.9 \pm 0.4$ & $7.2 \pm 0.1$ & $6.5 \pm 0.3$ & $9.3 \pm 0.5$ & $7.1 \pm 0.2$ & $6.0 \pm 0.2$ \\
\hline $\mathrm{AMH}, \mathrm{ng} / \mathrm{ml}$ & $1.29 \pm .37$ & $2.85 \pm .75$ & $7.72 \pm 2.6$ & $1.20 \pm .42$ & $3.04 \pm .70$ & $6.69 \pm 2.4$ & $0.99 \pm .52$ & $2.77 \pm .76$ & $6.81 \pm 1.4$ \\
\hline rFSH,IU & $2356 \pm 109$ & $1925 \pm 57$ & $1500 \pm 65$ & $2157 \pm 130$ & $1933 \pm 125$ & $1511 \pm 80$ & $4108 \pm 143$ & $2789 \pm 97$ & $1530 \pm 76$ \\
\hline AFC & $8(0-30)$ & $19(0-45)$ & $30(0-54)$ & $8(0-28)$ & $17(0-30)$ & $25(0-37)$ & $8(0-22)$ & $16(0-36)$ & $23(0-30)$ \\
\hline $\begin{array}{l}\text { No. of } \\
\text { oocytes } \\
\text { retrieved }\end{array}$ & $4(0-28)$ & $17(0-37)$ & $23(0-49)$ & $8(0-23)$ & $16(0-29)$ & $21(0-30)$ & $7(0-19)$ & $14(0-33)$ & $20(0-29)$ \\
\hline $\begin{array}{l}\text { No. of } \\
\text { 2PNs }\end{array}$ & $3(0-9)$ & $13(0-15)$ & $18(0-26)$ & $4(0-10)$ & $15(0-20)$ & $19(0-25)$ & $5(0-10)$ & $10(0-19)$ & $16(0-24)$ \\
\hline $\begin{array}{l}\text { No.of } \\
\text { blastocysts }\end{array}$ & 305 & 1175 & 1575 & 150 & 705 & 180 & 235 & 125 & 160 \\
\hline euploid, n & 215 & 665 & 875 & 105 & 535 & 110 & 215 & 100 & 110 \\
\hline CLBR,\% & 34 & 46 & 49 & 32 & 44 & 47 & 26 & 31 & 43 \\
\hline OPR,\% & 26 & 40 & 42 & 21 & 35 & 39 & 16 & 22 & 37 \\
\hline $\begin{array}{l}\text { Miscarriage } \\
\text { rate,\% }\end{array}$ & 12 & 10 & 6 & 13 & 11 & 7 & 14 & 12 & 10 \\
\hline
\end{tabular}

TABLE 1 Continued. 


\begin{tabular}{|lllllllll|}
\hline$P$-value & & & & & & & & \\
\hline L. vs M. & L. vs H. & M. vs H. & L. vs M. & L.vs H. & M. vs H. & L. vs M. & L.vs H. & M. vs H. \\
\hline- & - & - & - & - & - & - & - & - \\
\hline NS & NS & NS & NS & NS & NS & NS & NS & NS \\
\hline NS & NS & NS & NS & NS & NS & NS & NS & NS \\
\hline .000 & .000 & .000 & .000 & .001 & .001 & .000 & .000 & .000 \\
\hline .000 & .000 & .000 & .000 & .000 & .000 & .000 & .000 & .000 \\
\hline .000 & .000 & .000 & .000 & .001 & .000 & .001 & .000 & .000 \\
\hline .000 & .000 & .002 & .001 & .004 & .097 & .001 & .000 & .198 \\
\hline .000 & .000 & .001 & .002 & .005 & .136 & .000 & .001 & .263 \\
\hline .000 & .000 & .001 & .001 & .004 & .122 & .001 & .001 & .224 \\
\hline .038 & .000 & .004 & .192 & .093 & .356 & .007 & .017 & .550 \\
\hline .896 & .024 & .018 & .115 & .220 & .922 & .043 & .039 & .712 \\
\hline .005 & .002 & .0467 & .02 & .006 & .046 & .024 & $<.001$ & .005 \\
\hline .018 & .002 & .287 & .001 & .002 & .467 & .022 & .001 & .001 \\
\hline .014 & .011 & .011 & .019 & .102 & .330 & .024 & .031 & .054 \\
\hline
\end{tabular}

Note: Values are presented as median (range) or \%. AFC= antral follicle count; $\mathrm{CLBR}=$ cumulative live birth rate: OPR=ongoing pregnancy rate; $\mathrm{NS}=$ not statistically significant

Table 2

Univariate and multivariate regression analysis for number of euploid blastocysts

\begin{tabular}{|llllll|}
\hline Variable & Univariate R & $P$ value & Multivariate R & $95 \% \mathrm{Cl}$ & $P$ value \\
\hline Number of euploidy & & & & & \\
\hline Age, $\mathrm{y}$ & 0.230 & $<.001$ & -0.115 & $(-0.163,-0.067)$ & $<.001$ \\
\hline AMH, $\mathrm{ng} / \mathrm{ml}$ & 0.240 & $<.001$ & 0.147 & $(0.048,0.245)$ & 0.004 \\
\hline Oocytes retrieved, $\mathrm{n}$ & 0.531 & $<.001$ & 0.069 & $(0.037,0.102)$ & $<.001$ \\
\hline
\end{tabular}

Note: The complete multivariate model for total number euploid blastocyst is as follows: $y=4.099+0.147 * A M H-0.115^{\star}$ female age $+0.069 *$ the number of oocytes retrieved $\left(R^{2}=0.377\right.$; adjusted $R^{2}=0.367 ; F$ ratio $\left.=38.54, P<0.001\right)$ 
Table 3

A comparison of baseline demographic and cycle characteristics according to whether patients achieved birth after euploidy embryo transfer

\begin{tabular}{|c|c|c|c|}
\hline Variable & $\begin{array}{l}\text { Birth } \\
(n=515)(\%)\end{array}$ & $\begin{array}{l}\text { No birth } \\
(\mathrm{n}=460)(\%)\end{array}$ & $P$ value \\
\hline \multicolumn{4}{|l|}{ Age, y } \\
\hline$\leq 34$ & $305(59.2)$ & $185(40.2)$ & 0.013 \\
\hline $35-38$ & $120(23.3)$ & $120(26.1)$ & \\
\hline$\geq 39$ & $90(17.5)$ & 155(33.7) & \\
\hline $\mathrm{BMI}, \mathrm{kg} / \mathrm{m}^{2}$ & & & 0.453 \\
\hline$<24$ & $30(6)$ & $20(4)$ & \\
\hline $24-28$ & $350(68)$ & $350(76)$ & \\
\hline$>28$ & 135(26) & $90(20)$ & \\
\hline $\mathrm{AMH}, \mathrm{ng} / \mathrm{ml}$ & & & 0.001 \\
\hline$\leq 1.84$ & $85(17)$ & $160(35)$ & \\
\hline $1.84-4.52$ & $250(49)$ & $235(51)$ & \\
\hline$\geq 4.52$ & $180(34)$ & $65(14)$ & \\
\hline No. of oocytes retrieved, $n$ & & & 0.002 \\
\hline 5 & $35(7)$ & $35(8)$ & \\
\hline $5-10$ & $70(14)$ & $160(35)$ & \\
\hline 10 & $410(79)$ & $265(57)$ & \\
\hline $\mathrm{COH}$ cycles, $\mathrm{n}$ & $1.74 \pm 1.10$ & $1.90 \pm 1.11$ & 0.177 \\
\hline FET cycles, $\mathrm{n}$ & $1.99 \pm 1.43$ & $1.74 \pm 1.90$ & 0.030 \\
\hline Total blastocysts, $\mathrm{n}$ & $6.07 \pm 4.15$ & $3.85 \pm 3.19$ & $<.001$ \\
\hline Total euploid blastocysts, $n$ & $2.39 \pm 2.08$ & $0.93 \pm 1.78$ & $<.001$ \\
\hline Aneuploid blastocysts, $\mathrm{n}$ & $3.61 \pm 3.04$ & $2.87 \pm 2.27$ & 0.092 \\
\hline embryo transfer method, $\mathrm{n}$ & & & 0.585 \\
\hline natural cycle & $215(42$ & $200(43)$ & \\
\hline modified natural cycle & $300(58$ & $260(57)$ & \\
\hline Progesterone route, $\mathrm{n}$ & & & 0.098 \\
\hline Vaginal/oral & $35(7)$ & $20(4)$ & \\
\hline both & $480(93)$ & $440(96)$ & \\
\hline
\end{tabular}

\section{Supplementary Figure}

Supplementary Figure 1 is not available with this version.

Figures 


\begin{tabular}{|c|c|c|c|c|c|c|c|}
\hline Characteristics & Total & Live birth & $\begin{array}{c}\text { Non-live } \\
\text { birth }\end{array}$ & & Adjust & d OR( $95 \% \mathrm{CI})$ & $P$ Value \\
\hline age(y) & & & & & & & 0.013 \\
\hline$\leqslant 34$ & 490 & 305 & 185 & & & Reference & \\
\hline $35-38$ & 240 & 120 & 120 & & r & $1.91(0.88,4.16)$ & \\
\hline$\geqslant 39$ & 245 & 90 & 155 & & - & $1.39(0.58,3.30)$ & \\
\hline $\mathrm{AMH}(\mathrm{ng} / \mathrm{ml})$ & & & & & & & 0.001 \\
\hline$\leqslant 1.84$ & 245 & 85 & 160 & & & Reference & \\
\hline $1.84-4.52$ & 485 & 250 & 235 & $\sqrt{\mathbf{m}}-1$ & & $0.27(0.09,0.77)$ & \\
\hline$\geqslant 4.52$ & 245 & 180 & 65 & $r$ & & $0.48(0.22,1.04)$ & \\
\hline FET cycles(n) & 1825 & $1.99 \pm 1.43$ & $1.74 \pm 1.90$ & & $\vdash$ & $1.66(0.43,6.38)$ & 0.03 \\
\hline No. of Oocytes & & & & & & & 0.002 \\
\hline$\leqslant 5$ & 70 & 35 & 35 & & & Reference & \\
\hline $5 \sim 10$ & 230 & 70 & 160 & $r$ & & $0.49(0.22,1.10)$ & \\
\hline \multirow[t]{3}{*}{$\geqslant 10$} & 675 & 410 & 265 & 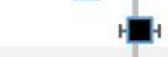 & & $1.05(0.87,1.25)$ & \\
\hline & & & & 0.00 & 2.00 & & \\
\hline & & & $\begin{array}{l}\text { Favor of } \\
\text { Non-live birth }\end{array}$ & & & Favor of Live birth & \\
\hline
\end{tabular}

Figure 1

Live Birth Outome 